\title{
Revising definitions of sepsis
}

\author{
Anne M. Drewry and Richard S. Hotchkiss \\ Department of Anesthesiology (A.M.D), Department of Anesthesiology (R.S.H.), Washington \\ University School of Medicine, 660 South Euclid, St. Louis, MO 63110, USA
}

\section{Standfirst}

The traditional definition of sepsis requires the presence of at least two systemic inflammatory response syndrome (SIRS) criteria in addition to a suspected or proven infection. A recent large retrospective study, however, suggests that the requirement for two SIRS criteria excludes one in eight patients with severe sepsis.

Sepsis is one of the most common causes of death in critically ill patients. ${ }^{1}$ It is also one of the most heterogeneous syndromes-in terms of its pathophysiology and symptomologyencountered in the intensive care unit. Despite its prevalence no standard diagnostic test has been developed to detect the onset of sepsis, and its diagnosis, which is primarily based on recognition of typical signs and symptoms, can therefore be challenging. In 1992, an international consensus conference convened to clarify the definitions of sepsis, severe sepsis, and septic shock to enable standardization of research protocols and to improve clinical detection. ${ }^{2}$ The resulting consensus report described the 'systemic inflammatory response syndrome (SIRS)' as the clinical response to an inflammatory process, requiring the presence of at least two of the following criteria for diagnosis: body temperature $>38^{\circ} \mathrm{C}$ or $\left\langle 36^{\circ} \mathrm{C}\right.$; heart rate $>90$ beats per minute; respiratory rate $>20$ breaths per minute or arterial partial pressure of carbon dioxide $\left(\mathrm{PaCO}_{2}\right)<32$ torr $(<4.3 \mathrm{kPa})$; or white blood cell count $>12,000$ cells $/ \mathrm{mm}^{3}$ or $<4,000$ cells $/ \mathrm{mm}^{3}$. 'Sepsis' was defined as a subgroup of SIRS when infection was determined to be the cause of the inflammatory process, and 'severe sepsis' was defined as organ dysfunction in the setting of sepsis. Since 1992, these definitions have served as the basis for enrollment criteria in major sepsis clinical trials as well as the backbone of the most commonly used sepsis screening tools.

Findings from a new retrospective study published in the New England Journal of Medicine, challenge these definitions. Although the 1992 definition of sepsis has previously been criticized as being too non-specific, Kaukonen et al. hypothesized that this definition would also have low sensitivity. ${ }^{3}$ In their retrospective study, Kaukonen et al. sought to quantify the validity and sensitivity of using the presence of at least two SIRS criteria to diagnose severe sepsis in critically ill patients. ${ }^{3}$ For the purposes of their study, the researchers redefined 'severe sepsis' as the failure of at least one organ (as defined by Sequential Organ Failure Assessment [SOFA] scores) in the setting of infection (defined by an admission

Correspondence to: A. M. D., drewrya @ anest.wustl.edu.

Competing interests

A.M.D. declares no competing interests. 
diagnostic code for infection). Of the 109,663 patients identified with organ failure and infection, 13,278 (12.1\%) did not meet the threshold of two SIRS criteria as required by the traditional definition of sepsis. Furthermore, although mortality was lower in the SIRSnegative patients ( $16.1 \%$ versus $24.5 \%$ ), it was not inconsequential.

These results are not entirely unexpected. Over the past 20 years, increased understanding of the pathophysiological mechanisms in sepsis has demonstrated that sepsis cannot be described simply as a syndrome of hyperinflammation. At the onset of sepsis, patients initiate both proinflammatory and anti-inflammatory responses, and many patients with sepsis demonstrate signs of impaired innate and cellular immunity. ${ }^{4}$ Predominance of a hyper-immune or hypo-immune response can vary between patients, depending on a number of host-specific and pathogen-specific factors, and can also vary within an individual throughout the course of their illness. ${ }^{5}$ Wide variation in the clinical syndrome between patients is therefore not surprising. This study's findings highlight the importance of recognizing that some infected patients, especially those who are elderly, may be less likely than others to demonstrate the symptoms of SIRS while remaining at considerable risk of organ failure and death.

For physicians treating patients with renal dysfunction, this work is particularly relevant. Patients requiring haemodialysis are at high risk of sepsis because of impaired immunity and the need for long-term vascular access. When these patients do develop sepsis, their response to pathogens can be blunted because of their inability to mount a vigorous immunologic defense. Frequently, septic patients on haemodialysis present with subtle clinical findings, such as decreased mental status, while lacking other SIRS criteria. A high index of suspicion for sepsis is therefore needed in these patients.

The most important interventions in sepsis-administration of appropriate antibiotics and goal-directed resuscitation - must occur early to maximize effectiveness. ${ }^{6,7}$ It is impossible to determine from the data presented by Kaukonen et al. whether SIRS-negative patients suffered increased morbidity or mortality due to delays in appropriate diagnosis or treatment of sepsis. In their study population, clinical diagnosis (or at least documentation) of sepsis did not seem inherently tied to the presence or absence of two SIRS criteria, given that $34 \%$ of SIRS-negative patients were given an admission diagnosis of sepsis whereas over half of the SIRS-positive infected patients were not. ${ }^{3}$ This inconsistency probably reflects the reality in most clinical settings. The most recent Surviving Sepsis Campaign guidelines published in 2012 define sepsis as "the presence (probably or documented) of infection together with systemic manifestations of infection,"; no specific emphasis is placed on the traditional SIRS criteria. ${ }^{8}$ Patients with infection and organ dysfunction (even those without two SIRS criteria) would meet this description; however, of sepsis screening tools and automated alert systems continue to require the presence of two SIRS criteria to diagnosis sepsis. ${ }^{9}$ As alert systems become more sophisticated, the accuracy of detecting sepsis may increase by identifying deviations in vital signs from baseline or changes in patterns of vital signs rather than relying on specific numerical cutoffs. ${ }^{10}$

Compared to clinical practice, the requirement for two SIRS criteria to diagnose sepsis is more stringent in clinical research where inclusion and exclusion criteria are rigorously 
applied. Recently, increased appreciation of the heterogeneity of sepsis had led to a paradigm shift in sepsis research, with greater emphasis on targeting therapeutic interventions to patients with specific pathophysiological abnormalities rather than to broad populations of patients with sepsis. Nevertheless, many sepsis trials still use the presence of two SIRS criteria to identify potentially eligible patients. Kaukonen et al. argue that strict adherence to this traditional definition of sepsis excludes one in eight patients with severe sepsis. ${ }^{3}$ It may therefore be time to abandon this old definition in favour of one that focuses on the presence of infection and organ dysfunction (Figure 1). Such a move would be especially pertinent to trials of therapeutic agents aimed at mechanisms that are more commonly seen in elderly patients (for example, immunosenescence), who are at high risk of developing organ dysfunction in the absence of two SIRS criteria.

\section{Acknowledgements}

A.M.D. is supported by the Foundation for Anesthesia Education and Research and the Washington University Institute of Clinical and Translational Sciences grant UL1 TR000448 from the National Center for Advancing Translational Sciences. R.S.H. is supported by National Institute of Health grants GM 44118 and GM 55194.

R.S.H. has received grant support from MedImmune, Bristol-Myers Squibb, GlaxoSmithKline, Agennix and Aurigene.

\section{References}

1. Brun-Buisson C, Meshaka P, Pinton P, Vallet B. EPISEPSIS: a reappraisal of the epidemiology and outcome of severe sepsis in French intensive care units. Intensive Care Med. 2004; 30:580-588. [PubMed: 14997295]

2. American College of Chest Physicians/Society of Critical Care Medicine Consensus Conference: Definitions for sepsis and organ failure and guidelines for the use of innovative therapies in sepsis. Critical Care Med. 1992; 20:864-874. [PubMed: 1597042]

3. Kaukonen K, Bailey M, Pilcher D, Cooper DJ, Bellomo R. Systemic inflammatory response syndrome criteria in defining severe sepsis. N Eng J Med. http://dx.doi.org/10.1056/ NEJMoa1415236.

4. Angus DC, Van der Poll T. Severe sepsis and septic shock. New Eng J Med. 2013; 369:840-851. [PubMed: 23984731]

5. Hotchkiss RS, Monneret G, Payen D. Immunosuppression in sepsis: a novel understanding of the disorder and a new therapeutic approach. Lancet Infect Dis. 2013; 13:260-268. [PubMed: 23427891]

6. Kumar A, et al. Duration of hypotension before initiation of effective antimicrobial therapy is the critical determinant of survival in septic shock. Crit Care Med. 2006; 35:1589-1596. [PubMed: 16625125]

7. Rivers E, et al. Early goal-directed therapy in the treatment of severe sepsis and septic shock. N Eng J Med. 2001; 345:1369-1377.

8. Dellinger RP, et al. Surviving sepsis campaign: International guidelines for management of severe sepsis and septic shock: 2012. Crit Care Med. 2013; 41:580-637. [PubMed: 23353941]

9. Makam AN, Nguyen OK, Auerback AD. Diagnostic accuracy and effectiveness of automated electronic sepsis alert systems: a systematic review. J Hosp Med. http://dx.doi.org/10.1002/jhm. 2347. Drewry AM, Fuller BM, Bailey TC, Hotchkiss RS. Body temperature patterns as a predictor of hospital-acquired sepsis in afebrile adult intensive care unit patients: a case-control study. Crit Care. 2013; 17:R200. [PubMed: 24028682] 


\section{Biographies}

Richard Hotchkiss is a Professor of Anesthesiology and Surgery at Washington University School of Medicine in St. Louis, MO. The focus of his research includes identifying mechanisms of cell death in sepsis and developing therapies to reverse sepsis-induced immunosuppression.

Anne Drewry is an Assistant Professor of Anesthesiology at Washington University School of Medicine in St. Louis, MO. Her primary research interest involves studying the role of body temperature abnormalities in the diagnosis and management of sepsis. 
a.

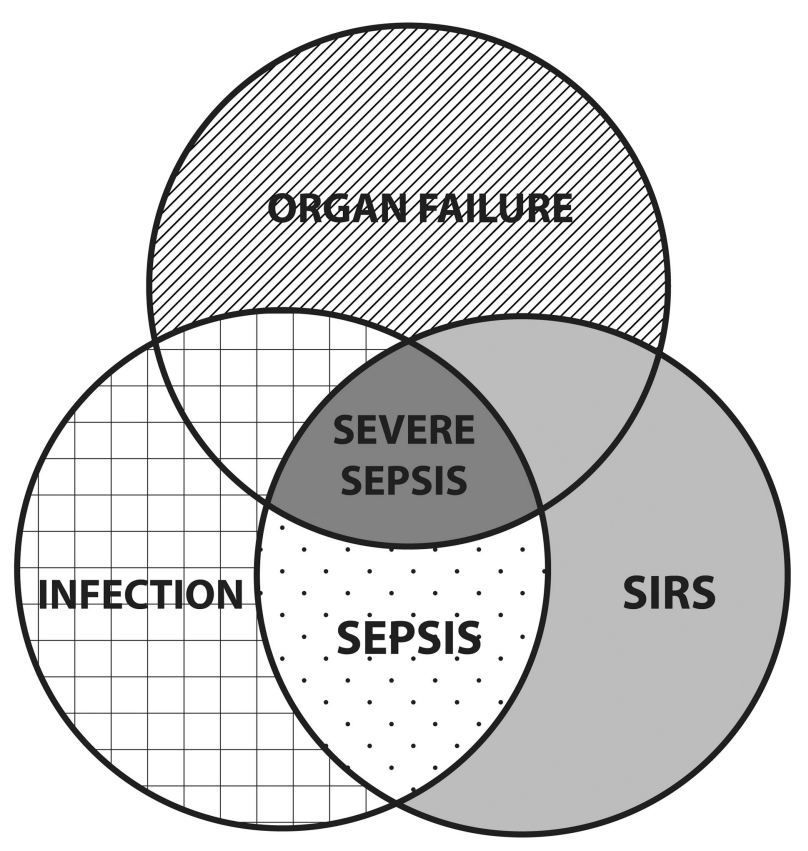

b.

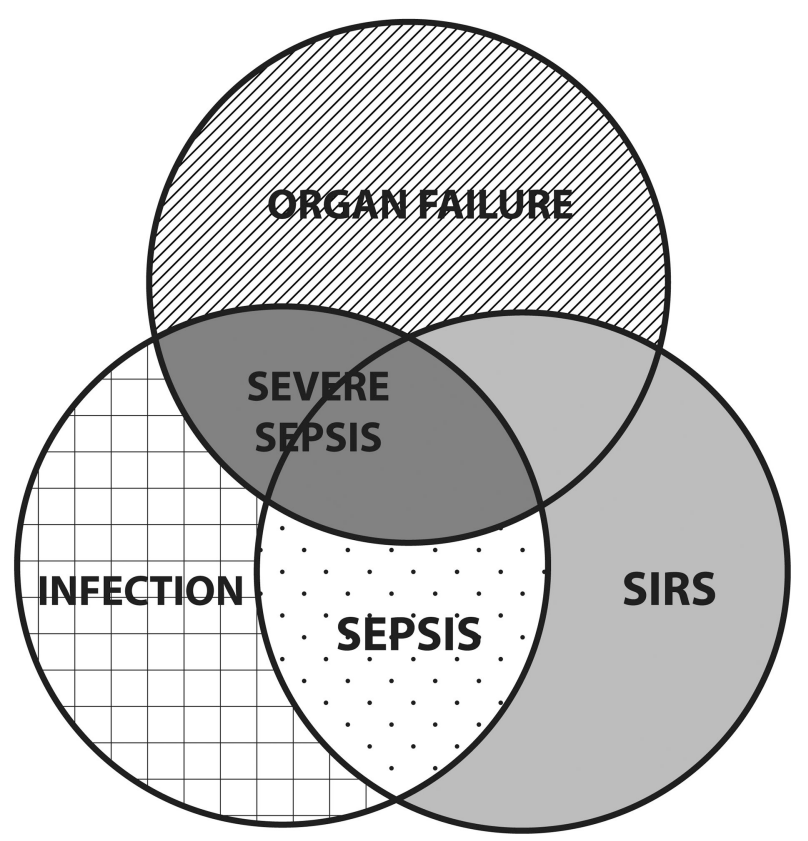

Figure 1.

Relationship between infection, SIRS, organ failure and severe sepsis. a The traditional model requires at least two SIRS criteria, infection and organ failure for a diagnosis of severe sepsis. b | A revised model allows for the presence of severe sepsis in the absence of SIRS criteria. Abbreviation: SIRS, systemic inflammatory response syndrome. 